\title{
MANUEL CASTELLS Y LA CUESTIÓN DEL PODER COMO CAPACIDAD: INVESTIGACIONES SISTEMÁTICAS, 1967-1982
}

\author{
Esteban Torres Castaños \\ Universidad Nacional de Córdoba, CIECS- CONICET, Argentina
}

\begin{abstract}
Resumen.- En el presente artículo proponemos una aproximación específica al modo en que los conceptos de poder y de capacidad se vinculan en las definiciones explícitas de poder de Manuel Castells en los textos de su periodo marxista, correspondientes a los años 1967-1982. A lo largo del trabajo intentaremos descubrir las operaciones teóricas principales, los movimientos de cambio conceptual, así como las concepciones del poder que el sociólogo catalán pone en juego a partir de esta relación específica. La investigación pone al descubierto la táctica de apropiación que despliega Castells en relación al concepto de poder de Poulantzas. El trabajo se inscribe en el marco de los esfuerzos actuales de revalorización de la teoría social y del poder, y de la tendencia del conjunto de las ciencias sociales a la recuperación del problema del poder como clave de interpretación general y como estrategia renovada de articulación disciplinaria.
\end{abstract}

Palabras claves.- Manuel Castells, poder, capacidad, teoría social

\begin{abstract}
In this article we propose a specific approximation to the way in which the concepts of power and capacity are linked in Manuel Castells' explicit definitions of power in his texts during his Marxist period, corresponding to the years $1967-1982$. Throughout the article, we will attempt to discover the main theoretical operations, conceptual change movements, as well as the conceptions of power that the Catalan sociologist brings into play from this specific relation. This piece of work reveals the appropriation strategy Castells displays in relation to Poulantzas' concept of power. This piece of work is in line with current efforts to revalue social theory and power, and the tendency in social science towards the recovery of the question of power as a key concept in general interpretation and as a renewed strategy of disciplinary articulation.
\end{abstract}

Key words.- Manuel Castells, power, capacity, social theory.

\section{INTRODUCCIÓN}

Nos propusimos estudiar la obra de Manuel Castells en el marco de una investigación de largo aliento sobre el concepto de poder en los teóricos sociales contemporáneos. En primer lugar, constatamos que toda pretensión actual de construir un marco de interpretación social del poder o de las relaciones de poder desde una perspectiva global y multidimensional que tome en consideración la expansión de las nuevas tecnologías de la información y la comunicación (TIC's), se encuentra inevitablemente con la obra del sociólogo español y su influencia en las discusiones y en las instituciones contemporáneas. El reconocimiento de la envergadura de la obra del autor es una práctica común en el campo de las ciencias sociales ${ }^{1}$, especialmente a partir de la publicación en inglés de su célebre

\footnotetext{
${ }^{1}$ Incluso los autores críticos consultados hacen referencia a Manuel Castells y su obra como "la versión más sofisticada de las teorías de la sociedad de la información" (Garnham, 2004), "la principal referencia mundial que señala el advenimiento de la era de la información" (Webster y
} 
trilogía de "La era de la información", entre el año 1996 y 1998, y luego traducida a 23 idiomas. Por otra parte, Castells es un autor institucional y políticamente muy influyente ${ }^{2}$.

En cuanto a la importancia analítica del concepto de poder, es menester recordar que es con la llegada de la modernidad que la categoría de poder se instala entre los conceptos centrales de la lógica y la arquitectónica de las filosofías y teorías políticas y sociales, y más importante aún, como parte insustituible y constitutiva del vocabulario social. En los términos de Koselleck, se trata de un "concepto fundamental" en tanto permite, a diferencia de los "conceptos en general", combinar experiencias y expectativas múltiples, de modo que resultan indispensables para la formulación de los problemas urgentes de la época (en Merlo, 1998: 94). Dado su carácter fundamental, en el concepto de poder, "se deposita la densa materialidad de una experiencia colectiva desplegada en el tiempo" (Chignola, 1998: 39). Por lo tanto, acordamos también con Giddens cuando afirma que "no existe un concepto más elemental que el de poder. El poder no puede ser asido, por así decir, después de formulados los conceptos mas básicas de la ciencia social" (Giddens, 1984: 309) ${ }^{3}$. Junto a ello, la recuperación del problema del poder como clave de interpretación general y como estrategia renovada de articulación disciplinaria pareciera constituirse en una de las lógicas de comunicación disponibles actualmente en las ciencias sociales para hacer frente a la creciente fragmentación disciplinaria.

Por otra parte, es necesario destacar el carácter original del trabajo propuesto. No registramos la existencia de investigaciones sistemáticas en curso que adopten como clave de análisis la pregunta por el poder de Castells. Sumado a ello, las reflexiones sobre la obra del autor a las que tuvimos acceso no abordan dicha categoría de forma sistemática, sino que se concentran por lo general en la relación que ésta establece con el problema clave de la soberanía estatal, en menor medida con la cuestión identitaria de los movimientos sociales, y sólo tangencialmente al tratar el problema del determinismo tecnológico ${ }^{4}$.

Blom, 2004), el "análisis más detallado sobre las nuevas tecnologías y el ascenso de la nueva sociedad en red" (Kellner, 2003), "una de las formulaciones más destacadas de las tesis relacionadas con la sociedad de la información" (Tremblay, 2003), "el ejemplo más acabado de descripción y definición conceptual de la sociedad que asoma entre las estructuras culturales del universo mediático" (Sierra Caballero, 2004), la mejor introducción a la interrelación entre las diferentes tendencias mundiales existentes en la actualidad (Van Dijk, 1999). Por otra parte, y de modo exagerado, Anthony Giddens y Chris Freeman han visto en Manuel Castells al Max Weber de nuestra época, al tiempo que Peter Hall compara la trilogía del autor con El Capital de Marx, y Touraine la señala como un futuro clásico del siglo XXI.

${ }^{2} \mathrm{Y}$ ello en varios aspectos que enriquecen la interpretación de sus textos. Si bien su obra es vasta, los tres volúmenes de la Era de la Información, representan una de las obras sociológicas que abordan la cuestión del poder más citadas y referenciadas en el mundo. Desde una óptica general, y a modo de ejemplo, el autor ocupa el cuarto lugar en el Índice de citas de Ciencias Sociales (SSCI) en el período 2000-2006 (1.- Anthony Giddens: 6190 citas; 2.- Robert Putnam: 4813; 3.- Jürgen Habermas: 4087; 4.- Manuel Castells: 3089 citas).

${ }^{3}$ La importancia del concepto de poder para la teoría social es señalada por el propio Castells, al afirmar que es necesario "poner los conflictos sociales, el cambio social, y el poder en el centro del análisis" (1998: 7). De igual modo, reaccionando de forma moderada a las doctrinas posmodernas, Castells nos insta en términos marxianos a "no olvidar otra vez la problemática del poder, el conflicto y la contradicción en juego" (1998: 7).

${ }^{4}$ Por una cuestión de espacio, obviaremos aquí las diferentes posiciones al respecto, así como las referencias bibliográficas específicas. 
Si bien trabajamos sobre el conjunto de la producción científica del sociólogo catalán, en el presente artículo nos concentramos exclusivamente en las relaciones que éste establece entre los conceptos de poder y de capacidad en las definiciones de poder que publicita en su período marxista ${ }^{5}$, que abarca desde los inicios de su carrera académica hasta el año $1982^{6}$.

Es evidente que la investigación sistemática sobre cierto concepto no se agota, y en ocasiones ni siquiera incluye, las definiciones que el autor propone de aquel. Menos aún la investigación socio-histórica de los conceptos, constitutiva de nuestro método general de trabajo (Torres Castaños, 2008), y que en sus aplicaciones más convencionales tiende a desconocer el valor de las definiciones. Ahora bien, ello no sucede en nuestra perspectiva, que tiene en el plano de la sistematización formal un punto de apoyo imprescindible. En este artículo trabajaremos exclusivamente sobre este último plano, asumiendo las limitaciones que dicho recorte conlleva ${ }^{7}$.

Dependiendo de la importancia teórica del autor, de su estrategia de escritura, y por supuesto de las formas de poner en juego su honestidad intelectual, a menudo los autores deciden no definir los conceptos con los cuales trabajan. Este no es el caso de Manuel Castells. Partiendo de dicha constatación, decidimos atender esta referencia en un primer momento. Ahora bien, ¿qué es lo que otorga valor analítico a una definición, y por tanto la hace merecedora de estudio? Apelando lo justo al sentido común, en principio diremos que representan una instancia que combina precisión, explicitación, concentración y abstracción de ideas, y donde cobra un valor especial la lógica de inclusión/exclusión de elementos, así como la forma que adopta la enunciación, como por ejemplo, el

\footnotetext{
${ }^{5}$ Junto al eje poder-capacidad, en la investigación general identificamos y luego desarrollamos otros siete ejes para el análisis de las definiciones de poder de Castells en este primer período: 1. Poder y toma del poder; 2 . Poder y dominación, 3. Poder y actores: las clases sociales; 4 . Poder e interés; 5. Poder "suma cero"; 6 . Poder y relación social, y finalmente 7. Poder y posición. En la búsqueda de transparentar nuestros problemas metodológicos, y por lo tanto abrir la caja negra de la investigación a nuestros lectores, consideramos necesario justificar, al menos en parte, el recorte establecido en este artículo: por cuestiones de extensión, es imposible ensayar cualquier tipo de desarrollo conjunto de todos los puntos en un sólo artículo. La condición para ello implicaría descartar los aspectos argumentativos del análisis, y dedicarse tan sólo a describir superficialmente cada uno de los ejes, así como esbozar a grandes rasgos sus interrelaciones. Descartamos absolutamente esta opción, ya que no reúne los requisitos analíticos y argumentativos de base de un producto de investigación conceptual y social. Lejos de cualquier solución ideal, optaremos por ir presentando cada uno de dichos ejes por separado, o al menos de alguno de ellos, en futuras publicaciones. Lógicamente, y más aún en lo que atañe a nuestros fines, el recorte no es gratuito: pagaremos el precio de no incluir las conclusiones generales del período considerado, que sólo se pueden extraer como síntesis del conjunto de los ejes. Ahora bien, junto a ello suponemos -lo cual también es necesario señalar- que los límites que impone dicho recorte de ningún modo sustraen el valor ni la validez al recorrido específico que aquí proponemos. Finalmente será el juicio de nuestros lectores el que dictamine hasta que punto es legítima nuestra elección.

6 Para analizar la definición del concepto de poder de Castells establecimos una periodización específica, dividida en tres momentos: 1967-1982; 1983-2003; 2004-2009. El cierre del último período es problemático, dado que el autor sigue activo, investigando y publicando en temas de poder. Por lo pronto, decidimos establecer el año 2009 como fecha de cierre de la última etapa para poder incluir su último libro individual "Comunicación y poder" (Ed. Alianza, Madrid, 2009).

${ }^{7}$ Las definiciones explícitas del poder representan tan sólo una referencia o indicador textual entre varios que empleamos para el análisis del concepto de poder, y en particular del concepto de Manuel Castells. Igualmente se trata de una referencia central, privilegiada, que por los motivos ya señalados merece ser estudiada en particular.
} 
orden de aparición de cada palabra o elemento, como eventual señal de su jerarquía o importancia. Aquí las definiciones de poder nos conducen principalmente $-y$ no únicamente- a la respuesta ofertada por el autor a una pregunta central: ¿Qué es el poder? Siendo que las definiciones no agotan la dimensión explicita del texto, y ésta última menos aún monopoliza la significación del concepto de poder, a partir de este artículo no podremos agotar la respuesta general a dicha la pregunta. Por el momento, nos contentaremos si logramos ingresar satisfactoriamente en ella.

Suponemos que la importancia crucial del poder para Castells, y en particular de la definición del poder, que es su expresión más abstracta y manifiesta, en parte se evidencia a partir de dos hechos que en este artículo no comprobaremos. El primero de ellos es la cantidad, el tipo y la envergadura de las modificaciones que ha registrado la definición del concepto a lo largo de su obra, y que en algunos casos acompañan los cambios de su teoría y epistemología social. El segundo acontecimiento son las propias declaraciones metodológicas del autor, siempre a favor de la centralidad del análisis del poder (y del cambio social) para la investigación social.

A partir de aquí, y luego de establecer las especificaciones bibliográficas de rigor, iniciaremos el proceso de análisis con la esperanza de aproximarnos al objetivo central que perseguimos en el artículo, y que tiende a concretarse en tres aspectos diferenciables e interrelacionados: el descubrimiento de las operaciones teóricas principales, los movimientos de cambio conceptual y las concepciones del poder involucradas en el vínculo que nuestro autor establece entre los conceptos de poder y de capacidad en sus definiciones de poder.

\section{PRECISIONES BIBLIOGRÁFICAS}

Las definiciones de poder en este primer período se registran en tres libros, correspondientes a los años 1971, 1973 y 1974 respectivamente, y dos capítulos de libro, el primero de 1969 y el restante de $1973^{8}$. Efectuada la delimitación, quedan excluidos de este punto 14 libros (10 individuales), de los cuales seis tratan aspectos del poder (y que a partir de aquí llamaremos textos o materiales "específicos"), 13 capítulos de libros (ocho específicos), más de 30 artículos $^{9}$, así como un par de informes de investigación, todos publicados durante el transcurso de este primer ciclo, y que luego analizaremos en futuros artículos, a medida que

\footnotetext{
${ }^{8}$ Los textos de Castells que explicitan una definición de poder, y que aquí analizamos en particular, son los siguientes: Libros: Problemas de investigación en sociología urbana (1971a); La cuestión urbana (1972a); La lucha de clases en Chile (1974a). Artículos: "Theoretical Proposition for an Experimental Study of Urban Social Movements" (1969a), y "Comentario: La teoría marxista de las clases sociales y la lucha de clases en América Latina" (1973c). Constatamos que ninguno de los textos de Castells incluidos en la selección son en coautoría.

${ }^{9}$ Los referimos a los siguientes libros (individuales, en co-autoría y editados o co-editados): 1968a; 1973a; 1973b; 1974b; 1974c; 1975a; 1975b; 1976a; 1977a; 1978a; 1978b; 1978c; 1981a; 1981b. Capítulos de libros: 1968b; 1969b; 1969c; 1973d; 1975c; 1975d; 1976b; 1976c; 1976d; 1977b; 1981c; 1982a; 1982b. Artículos: 1967; 1968c; 1969d; 1969e; 1969f; 1970a; 1970b; 1970c; 1971b; 1971c; 1972b; 1972c; 1972d; 1972e; 1973e; 1973f; 1973g; 1974d; 1974e; 1975e; 1975f; 1976e; 1976f; 1976g; 1977c; 1977d; 1977e; 1977f; 1978d; 1978e; 1979a; 1979b; 1980; 1981c; 1982c). Informes de investigación: 1981d; 1981e. Las referencias que están en cursiva corresponden a los textos específicos.
} 
se proyecten nuevas interrogaciones sobre el poder. De los materiales seleccionados en este período, se destacan dos libros claves: La cuestión urbana (1972) y Lucha de clases en Chile (1974). Mientras que el primero es sin dudas el texto más reconocido del período marxista del autor, consideramos que el segundo es su texto más atento al poder, y junto a ello, su producción más "política", escrita, según expresiones del propio autor, al calor de la derrota de la Unidad popular en Chile, del golpe de Estado y del asesinato de Salvador Allende. Siendo que el período que decidimos establecer transcurre entre los años 1969 que es cuando el autor comienza a publicar- y 1982, podemos observar que los textos se concentran en lo que llamaremos un ciclo de tiempo corto, de cinco años, a principios del periodo. El final de este ciclo corto abre un período de silencio respecto al poder y sus definiciones, que se extiende entre 1975 y 1982. En próximas publicaciones intentaremos sumar elementos que permitan esclarecer, al menos en algún punto, los motivos e implicancias que aquí se ponen en juego.

\section{PODER Y CAPACIDAD}

\subsection{Las definiciones concretas}

En el marco de este primer período registramos nueve definiciones de poder. Del total, tres son indirectas ${ }^{10}$. Tal como señalamos al inicio del capítulo, las definiciones de poder se expresan en un marco bibliográfico y temporal acotado: hablamos de dos libros y dos capítulos de libros, publicados entre el año 1969 y 1974. De las nueve definiciones registradas, dos aparecen en La cuestión urbana y cinco en Lucha de clases en Chile, transformando a éste último en el libro de mayores referencias del período.

Aquí analizaremos las diferentes definiciones de poder, así como la relación entre ellas, a partir de la desagregación de los elementos centrales que las componen. Como venimos repitiendo, en este caso puntual nos concentraremos en la relación entre poder y capacidad. Dado que cada elemento se analiza "en" y "entre" las definiciones, ello implica, en cada caso, la necesidad de volver una y otra vez sobre las mismas definiciones. Para intentar simplificar el entramado del análisis, evitando la repetición acumulativa de las mismas citas (lo que a su vez engrosaría el trabajo en forma desmedida), optamos aquí, en un primer momento, por la presentación cronológica de la totalidad de las definiciones, con sus referencias bibliográficas básicas, adjudicándoles un código de referencia a cada una. Luego, a partir del siguiente punto, nos adentraremos en el análisis de cada elemento. Una vez allí, cada vez que la situación lo exija, optaremos por priorizar el código de la definición a la propia cita textual. Por supuesto, el análisis de cada elemento contempla el ingreso de nuevas referencias bibliográficas. A partir de este modo de exposición esperamos contribuir a la mejor lectura y comprensión del apartado, a sabiendas que será necesario contar con la voluntad del lector para eventualmente retornar a estas referencias iniciales. A favor de este modo

\footnotetext{
10 Por "indirectas" entendemos que no aluden directamente al concepto de poder sino a un concepto semejante que en este caso se definiría de igual modo, o bien tendería a expresar el concepto de poder en un nivel de abstracción diferente. El fundamento de su carácter indirecto lo aclararemos en cada caso.
} 
de exposición, reconocemos que el lector podrá, sin mayor esfuerzo, identificar relaciones importantes que posiblemente hayamos descuidado. Veamos entonces:

Definición 1 (d1). Aparecida en el capítulo de libro "Theoretical Proposition for an Experimental Study of Urban Social Movements", publicado durante el año 1969:

The political refers to the structures by which a societies exercises control over the different instantes which constitute it, thereby assuring the domination of a particular social class relations.

Politics refers to the system of power relations. The theoretical location of the concept of power is that of class relations. By power we mean the capacity of one social class to realice its specific objective interests at the expense of others (Castells, 1969a: 148).

Definición 2 (d2). En el libro La cuestión urbana, año 1972:

Definimos las relaciones de poder como relaciones entre clases sociales y las clases sociales como combinaciones de lugares contradictorios definidos en el conjunto de la estructura social, concibiendo al poder como la capacidad de una clase o fracción de clase para realizar sus intereses objetivos, a expensas de las clases, o conjunto de clases, contradictorias, con quienes están en contradicción ${ }^{11}$ (1972a: 289).

Definición 3 (d3). Nuevamente en La cuestión urbana:

- Lo político designa la instancia por la cual una sociedad trata las contradicciones y desniveles de las diferentes instancias que la componen y reproduce, ampliándolas, las leyes estructurales, asegurando asi la relación de los intereses de la clase social dominante.

- La política designa el sistema de relaciones de poder. El lugar teórico del concepto de poder es el de las relaciones de clase. Se entiende por poder la capacidad de una clase social para realizar sus intereses objetivos específicos a expensas de las otras. Por intereses objetivos entendemos el predominio de los elementos estructurales (que definen, por su combinación, una clase) sobre los otros elementos que están en contradicción (1972a: 309).

Definición 4 (d4). En el capítulo de libro La teoría marxista de las clases sociales y la lucha de clases en América Latina, del año 1973. En este caso se trata de una definición indirecta, ya que se ocupa del concepto de posición de clase. Integramos la definición a partir de constatar que poder y posición de clase son en cierto modo equiparables:

Si la posición de clase se define por la capacidad estructural de determinar la organización social en función de sus intereses específicos, la identificación de la pertenencia de clase de una práctica podrá hacerse por el análisis de su horizonte estructural, es decir, por la imputación de los efectos que tiende a producir objetivamente en la formación social en cuestión. Y decimos que tiende porque toda práctica se encuentra confrontada a otras prácticas en su conjunto el que en último término actuará efectivamente sobre las relaciones sociales existentes (1973c: 169).

\footnotetext{
11 "Véase Nicos Poulantzas, Poder político y clases sociales, Siglo XXI, México, 1970" (Pié de página de Castells).
} 
Definición 5 (d5). En el libro Lucha de clases en Chile, publicado en el año 1974:

Puesto que, como es sabido, el poder no es un "objeto", un "atributo", una entidad material que se pueda apropiar, sino una relación social, una capacidad de realizar los intereses de clase... Pero tal situación no puede desorientar sino a quienes desligan el análisis del poder del análisis de los intereses de las clases en lucha. En cambio, si se parte de la estrecha relación entre unos y otros, si el poder no es "dominación", sino dominación para realizar intereses objetivos anclados en la estructura económica, entonces la respuesta puede ser dada a través del análisis de la lucha de clases en las principales contradicciones que caracterizan una sociedad, en particular en aquellas relativas a las relaciones de producción y a la apropiación del producto por ellas determinada. O sea, que en lugar de tratar la cuestión del poder a nivel de la autoidentificación de clase que hacen los aparatos políticos o las ideologías de los grupos sociales, es necesario examinarla a través del grado de desarrollo de los intereses objetivos de clase en las prácticas de lucha en los diferentes frentes, económico, político e ideológico... Ahora bien, ¿sobre qué intereses?, sobre sus intereses económicos, pero a largo plazo, es decir, en concreto, sobre la capacidad política de cada clase para realizar sus intereses económicos estructurales (1974a: 151).

Definición 6 (d6). En Lucha de clases en Chile:

"La expresión concentrada de la lucha de clases es la lucha política de clases, o sea aquella que tiene por objetivo el poder. Por poder no puede entenderse una simple atribución de la capacidad coercitiva, como lo quiere la teoría liberal y la histórica del poder, sino la capacidad de organizar el conjunto de la sociedad en torno a la realización de los intereses específicos de una clase social y de su fracción hegemónica ${ }^{12}$ lo cual sólo puede hacerse a expensas de los intereses de otras clases, aquellas con las cuales se está en contradicción, por el lugar recíproco y antagónico ocupado, en primer término, en las declaraciones de producción" (1974a: 361).

Definición 7 (d7). En el mismo libro:

El poder no es un objeto sino una relación social. Una relación entre las clases y que tiene por referencia la organización del conjunto de la sociedad en función de sus intereses. Por tanto, de lo que se puede hablar es de las posiciones que se van ganando en el combate, de los cambios de las relaciones de poder, de la correlación de fuerzas más o menos favorable. La dualidad de poderes no es tal, sino la expresión de una correlación tan favorable a la clase obrera que esta puede generar sus propios aparatos de poder en las masas y en el conjunto de la sociedad en contradicción con el estado burgués, simultáneamente desmantelado, en una correspondencia recíproca y de sentido inverso entre la capacidad política del movimiento popular y la de la burguesía (1974a: 397)

Definición 8 (d8). En el mismo libro. Aquí presentamos la segunda definición indirecta, centrada en el concepto de toma del poder. No se trata exactamente de cierta equiparación con el poder, tal como sucede en la d4, sino de la toma del poder como una instancia de concreción del concepto de poder:

\footnotetext{
12 "Véase Nicos Poulantzas, ob.cit" (Pié de página de Castells).
} 
Es decir que la toma del poder por una clase (siempre a través de un bloque de clases en el que se asegure políticamente su hegemonía) ${ }^{13}$ consiste en la capacidad de organizar las relaciones de propiedad, las relaciones sociales de producción y, a partir de ellas, el conjunto de relaciones, procesos y aparatos económicos, políticos e ideológicos, en forma tal que aseguren progresivamente, cada vez de manera más nítida, la plena realización de sus intereses específicos" (1974a: 361).

Definición 9 (d9). En el mismo libro. Aquí exponemos la tercera y última definición indirecta, nuevamente centrada en el concepto de toma del poder, aunque en un nivel de mayor concreción que el anterior:

Por un lado, puesto que el aparato del Estado resume-condensa el poder político de las clases dominantes, puesto que a través de él se organiza, regula, integra, reprime, el conjunto de relaciones sociales, la toma del poder consiste en la destrucción del aparato del Estado ligado a las clases dominantes y en la construcción de uno nuevo, expresión de la nueva dominación de clase, es decir de la dictadura del proletariado, tal como anteriormente se ejercía, bajo distintos regimenes, la dictadura de la burguesía (1974a: 363).

Para Manuel Castells, en este primer período, todo poder es poder de clase. Del mismo modo, toda dominación es dominación de clase, todo interés es interés de clase, toda lucha es lucha de clases, y así un largo etcétera, que en las definiciones de poder incluye conceptos como los de capacidad (d1, d2, d3, d4, d5, d6 y d8) posición (d4 y d7) y pertenencia (d4). Las clases sociales, en la acepción marxista del autor, son los actores fundamentales y casi excluyentes del poder y la sociedad. Tanto la definición del poder como el conjunto del andamiaje conceptual que aquí edifican su sistematización general, se sustentan en gran medida en los aportes teóricos de Nicos Poulantzas. Respecto a éste, Castells despliega una táctica de apropiación cuando menos inquietante, que descubriremos gradualmente para no marear al lector.

\subsection{Poder y capacidad}

En este primer periodo, a partir de Poulantzas, Castells define el poder, en primera instancia, como una capacidad. Para nuestro autor, el poder es capacidad de clase. De las nueve definiciones listadas, siete se asocian directamente al concepto de capacidad. Así como Castells opta por aclarar la noción de clase social en ciertas definiciones de poder, no sucede lo mismo con la noción de capacidad. No provee respuestas a la pregunta ¿qué es una capacidad? Pareciera que ésta última opera en segunda instancia, exclusivamente como elemento para precisar el concepto de poder. Pero no por ello es menos importante. En el caso de Poulantzas es diferente: como veremos en el próximo punto, luego de reconocer la noción de capacidad como elemento del concepto de poder $^{14}$, éste se detiene en el análisis de la identificación entre capacidad y

\footnotetext{
13 "Según las tesis clásicas desarrolladas por Gramsci (Consultadas en Oeuvres Choisies, Editions Sociales, París)" (Pié de página de Castells). Aquí el autor no ofrece mayores precisiones sobre el uso y la apropiación de la perspectiva de Gramsci.

${ }^{14}$ Es importante recordar que la definición de poder que propone Poulantzas en 1968, en su versión restringida, es exactamente la misma que luego reproducirá Castells: "Puede intentarse, partiendo de estas observaciones, proponer un concepto de poder: se designará por poder la
} 
organización. Por su parte, como apuntaremos en breve, Foucault también se ocupa de explicitar la noción de capacidad en relación con el concepto de poder. Dado que tanto para Poulantzas como luego para Castells el poder se define en primera instancia a partir del concepto de capacidad, los movimientos de dicha noción en relación con la definición de poder, son siempre importantes para ésta última. Incluso podríamos señalar que en el concepto de capacidad de Castells, en sus formulaciones compuestas (cuando se acompaña de otra palabra que la precisa), se asientan las huellas de ciertos debates fundamentales de la teoría del poder, como aquellos que giran en torno a la naturaleza coercitiva o no del poder. Precisaremos estos comentarios en próximos apartados.

\subsubsection{Intereses y organización social}

A un nivel general, Castells emplea durante este primer período dos nociones de capacidad para definir el poder: la capacidad de realización de intereses y la capacidad de organización social. Cualquiera de ellas modifica sustancialmente la referencia dada por Poulantzas, que tiende a definir la capacidad como organización (y no capacidad de organización). Repasemos primero cada una de las acepciones de Castells:

a) El poder como capacidad de realización de intereses: Así se presenta en la d1, d2, d3 y d5. En el caso de la d1, d2 y d3, ésta se precisa como capacidad de realización de intereses a expensas de los otros. Ahora bien, ¿en qué consiste la "capacidad de realización" de las clases que conforman el poder? Si bien en todos los casos el objeto de la capacidad de realización son los intereses de clase, Castells no precisa lo que entiende exactamente por dicha capacidad en sí misma. Por ejemplo, ¿se trata de una capacidad más próxima a la práctica o a la posición de clase? ¿Hay un concepto de capacidad propio de las prácticas de clase, o la capacidad es en todo momento y exclusivamente un reconocimiento posicional? Luego, ¿de qué prácticas y/o posiciones estaríamos hablando? En principio, atendiendo a las precisiones de las definiciones mencionadas, el poder sería capacidad de realización de los intereses de cualquier clase a expensas de la otra. En este caso, tanto la clase dominante como la clase dominada tendrían poder. Si efectivamente es así, si ambas clases tienen capacidad de realización de intereses, al menos circunstancialmente, parecieran asomarse ciertos problemas de coherencia conceptual, ya que en algún punto atenta contra las delimitaciones previas de poder que venimos estableciendo. Veamos, ¿la dominación también se podría entender como capacidad de realización de clase? Esto es importante de definir, ya que hasta aquí dominación y poder, en sus acepciones dominantes, difieren sustancialmente: el concepto de poder, tal como lo venimos delimitando, se opone en varias ocasiones a la dominación, que mayoritariamente expresa la práctica institucional y/o estatal de las clases dominantes. Por lo tanto, el poder es una expresión particular, al igual que la dominación. Según los caminos conceptuales que venimos recorriendo, no toda práctica de clase es una práctica de poder, sino sólo aquellas que se identifican con las lógicas de cambio social, con la producción de efectos novedosos en la estructura social.

capacidad de una clase social para realizar sus intereses objetivos específicos" (Poulantzas, 1968: 124). 
b) El poder como capacidad de organización social: Se registra en la d4, d6, d8. Si bien todas responden a la capacidad señalada, éstas se manifiestan con leves diferencias en cada caso: capacidad estructural de determinar la organización social $\left(\mathrm{d} 4^{15}\right.$ ); capacidad de organizar el conjunto de la sociedad (d6); capacidad de organizar el conjunto de relaciones, procesos y aparatos económicos, políticos e ideológicos (d8). A ello eventualmente podemos sumar la d7, que si bien no hace referencia explicita al poder como capacidad sino como relación, se define a partir de los mismos parámetros organizativos: la organización del conjunto de la sociedad (d7).

Aquí es importante señalar que la capacidad de organización social, es capacidad de organización social para la realización de intereses, con lo cual integra buena parte de los elementos de la primera definición. Por otra parte, Castells tiende a identificar el poder como capacidad de organización, en oposición y/o como formula superadora de la noción de poder como "simple atribución de capacidad coercitiva" (d6). Al asumir el rechazo del poder como simple atribución de la capacidad coercitiva, ¿qué lugar le asigna a la coerción o la violencia en la noción de capacidad de organización? ¿Cómo se resuelve finalmente la tensión entre capacidad organizativa y capacidad coercitiva? Si bien todo indica que la primera se integra en la segunda, Castells no precisa el modo de participación de la coerción en la nueva formula organizacional del poder.

Asociado a esta segunda noción, en la d4 (que como sabemos alude al concepto de posición de clase y no directamente al de poder) aparece la referencia a un atributo novedoso de la capacidad, que luego no se repite en ninguna de las demás definiciones: la capacidad estructural, esto es, la explicitación de la dimensión estructural de la capacidad. Castells señala que la posición de clase se define por la capacidad estructural de organización social. En el caso que posición de clase y poder sean prácticamente sinónimos (lo cual por el momento es una hipótesis), ¿entonces porqué Castells no define el poder, en las restantes definiciones, como capacidad estructural y no tan solo como capacidad? Podemos deducir también que capacidad de una clase es capacidad estructural, lo cual reconfirma que la clase es una categoría estructural. Ahora bien, la otra posibilidad es que el poder no se defina exclusivamente a partir de la posición de clase, sino en un sentido más amplio, a partir de la posición de clase y la práctica de clase, en la primera como capacidad estructural y en la segunda a partir de la imputación de efectos de la práctica. Vemos que ambos elementos se hacen presentes y se distinguen en la d4. Si fuese así, ¿cómo se denomina la capacidad que no sería estructural? ¿Si es que hay poder en la práctica de clase, también es expresión de cierta capacidad? ¿Todo poder es capacidad? Tampoco encontramos en Castells la respuesta a estas preguntas.

Distanciados de cualquier coincidencia temporal en el uso de ambas nociones, lo que registramos es un movimiento de paso de la primera noción a la segunda: del poder como capacidad de realización de intereses específicos, mayoritariamente presente en La cuestión urbana, al poder como capacidad de organización social para la realización de intereses específicos, principalmente expresado en Lucha de clases en Chile. Ahora bien, ¿a que se debe la incursión de la idea de la

${ }^{15}$ En el caso de corroborar la equiparación de posición de clase y poder de clase, en la d4 aparecería por primera el poder ligado a la capacidad de organización del conjunto. 
organización social en la definición del poder? En un principio, pareciera que a partir de ello Castells suscribe a un concepto más amplio de poder, que encierra elementos constructivos, priorizando -como ya hemos señalado- la idea de capacidad organizativa a la de capacidad coercitiva. ¿En que medida involucra el poder como capacidad de organización social al Estado? ¿El cambio de la primera noción de capacidad a la segunda tiene que ver con un nuevo reconocimiento de la centralidad del Estado en el desarrollo de las capacidades de organización social de las clases sociales? Castells guarda silencio respecto a estas preguntas. Entre los posicionamientos sustantivos del autor en este primer periodo, es importante destacar el rechazo a la identificación plena entre poder y coerción, que el autor reconoce como una limitación de las teorías liberal e histórica del poder. Aquí Castells critica la perspectiva que suscribirá en el futuro.

El registro clave que aquí resta introducir, y que mencionamos al iniciar este punto, es la noción de capacidad de Poulantzas que acompaña su definición de poder. Esta se asemeja en cierto punto a la segunda acepción del vínculo poder capacidad de Castells, pero a diferencia de ésta, Poulantzas define la capacidad de clase directamente como organización de clase, para luego detenerse en su explicación:

El concepto de poder se refiere a la capacidad de una clase para realizar intereses objetivos específicos. Este elemento del concepto de poder se refiere, más particularmente, a los estudios de Marx y Lenin relativos a la organización de clase.... En ese sentido amplio, la palabra organización comprende simplemente las condiciones de una práctica de clase con "efectos pertinentes". Sin embargo, la teoría de la organización, en el sentido estricto de la palabra, en Marx, y sobre todo en Lenin, no comprende simplemente las prácticas de clase, las condiciones de existencia de clase en cuanto clase distinta -fuerza social-, sino las condiciones de poder de clase, es decir, las condiciones de una práctica que conduce a un poder de clase (Poulantzas, 1968: 128-129).

Mientras Castells, en su segunda acepción, concibe el poder como capacidad de organización social, aquí Poulantzas define el poder como capacidad de realización de intereses (al igual que la primera acepción de nuestro autor), pero luego agrega la definición de la capacidad como organización social. Constatada la diferencia, es necesario preguntar: ¿qué entiende Castells por capacidad, siendo que en su literalidad no equivale a organización social? Con ello se haría evidente un principio de redundancia en Castells ${ }^{16}$, incrementando de este modo la opacidad que acompaña su definición de poder. Por otra parte, aquí aparece un elemento crítico que se omite en las definiciones de Castells, y que sin dudas colabora en el entendimiento de la relación entre poder y capacidad. En el marco de la identificación entre capacidad y organización social, en la cita anterior Poulantzas define la organización social como condición de poder. Extrapolando la asociación, ¿podríamos concebir la capacidad -como un todo- como condición de poder? En la cita anterior se superponen dos elementos: por un lado el poder, que en principio se define en sentido estricto en relación a las "prácticas de clase con efecto pertinente", y por otra la capacidad y la organización social, que aparecen como condición de las prácticas de clase, luego como condición de

${ }^{16}$ Si bien no es el objetivo preciso de nuestro trabajo, siempre es difícil ampliar el análisis comparativo de las diferencias entre Castells y Poulantzas, ya que el primero no argumenta sus elecciones conceptuales al mismo nivel que Poulantzas. 
poder de clase, pero asumiendo finalmente que las condiciones de poder conducen al poder de clase. Las dudas aparecen a partir de la consideración, tanto de Poulantzas como de Castells, del poder como capacidad-organización. En el razonamiento que desplegamos hasta aquí, esta última identificación dejaría en cierta forma afuera a las prácticas de clase, dado que la capacidad se define como organización y ésta última es condición de las prácticas de clase, pero no define las prácticas en sí. Algunas páginas más adelante, Poulantzas amplía su definición del poder en relación al vínculo entre prácticas y organización de clase:

Más, por otra parte, si esa organización específica de una clase es la condición necesaria de su poder, no por eso es la condición suficiente. Esta observación nos permite ver mejor las razones de la distinción entre la práctica con "efectos pertinentes" de una clase y su organización de poder. La organización de poder de una clase no basta para su poder, porque, en primer lugar, ese poder se obtiene en los límites, en cuanto efecto, de las estructuras en el campo de las prácticas: el contrario de una concepción "voluntarista", puede verse que la realización efectiva de los intereses depende de esos límites. Hay también, sin embargo, otra razón que nos revela, por otra parte, el fundamento de la distinción entre la práctica con "efectos pertinentes" y la organización de poder: el concepto de poder especifica los efectos, como límites, de la estructura en las relaciones de las diversas prácticas de las clases en lucha. En este sentido, el poder indica relaciones no directamente determinada por estructura, y depende de la relación exacta de las fuerzas sociales presentes en la lucha de clases (Poulantzas, 1968: 131).

Si bien en esta segunda cita Poulantzas avanza en la especificación sobre la relación entre poder, prácticas y organización de clase, lo cual Castells en ningún momento realiza, no integra en la reflexión al concepto de capacidad, que rige en primera instancia su definición estricta de poder. Por lo tanto quedan dudas si finalmente la capacidad integra tanto a las prácticas y sus efectos como a la organización social, al poder y las condiciones de poder, o bien se circunscribe a los aspectos de organización, con lo cual su definición estricta de poder como capacidad de realización de intereses resultaría en cierto modo insatisfactoria. Esta última sería una definición circunscrita a lo que Poulantzas llama las "condiciones necesarias" del poder, renunciando a la representación de las "condiciones suficientes", que es precisamente a donde debe conducir una definición general de poder o de poder de clase. Igualmente, constatamos que la relación entre poder y capacidad integra uno de los ejes fundamentales de la teoría del poder de Castells, que apenas se explicita en este período, pero que tenderá a acentuarse en los próximos: la relación entre poder y organización.

\subsubsection{Capacidad y relación social}

La operación teórica central que realiza Castells en las definiciones de poder en relación a la noción de capacidad, es la eventual igualación de los conceptos de capacidad y relación social, o mejor dicho, entre poder, capacidad y relación social. Ésta se manifiesta de modo directo e indirecto. De forma directa, en la d5, cuando define al poder como "...una relación social, una capacidad de realizar los intereses de clase..." ${ }^{17}$ (1974a: 151). Y luego de modo indirecto, en la d1, d2, d3,

\footnotetext{
${ }^{17}$ Al interior de la cita las cursivas son del autor.
} 
cuando inmediatamente después de indicar que el lugar teórico del concepto de poder es la relación de clase, define al poder como capacidad de clase. A partir de ello, ¿podríamos decir que coexisten en las definiciones de poder de Castells de este primer período, una relación de igualación entre poder, capacidad y posición, al mismo tiempo que un vínculo de igualación entre poder, capacidad y relación social? Respecto a esta última, ¿qué vínculo entabla con la dialéctica? ¿Por qué otorgar status de operación teórica a la igualación entre capacidad y relación social? Aquí sólo responderemos a esta última: en resumidas cuentas, porque da cuenta de una resolución conceptual muy importante, en este caso desacertada: la noción de capacidad, a diferencia del concepto de relación social, no es habitualmente una expresión dialógica, en cualquiera de sus versiones (individual, organizacional, social, etc.), y no involucra necesariamente más de un actor. Si bien es una referencia extemporánea respecto al periodo en cuestión, nos tomamos la licencia para señalar que el propio Foucault, en 1983, hizo precisamente hincapié en la necesidad de diferenciar ambos elementos, basándose en argumentos similares a los nuestros:

En lo que concierne a este poder, en primera instancia es necesario distinguir aquel que se ejerce sobre las cosas y da a su vez la habilidad de modificar, usar, consumir y destruirlas -un poder que procede de aptitudes directamente inherentes al cuerpo o "apoyadas" en instrumentos externos. Diría que aquí hay una cuestión de "capacidad". Por otro lado lo que caracteriza al poder que estamos analizando es que este pone en juego las relaciones entre los individuos (o entre grupos). Para no engañarnos a nosotros mismos, si hablamos de las estructuras o los mecanismos del poder, es sólo en tanto suponemos que ciertas personas ejercen el poder sobre otros. El término "poder" designa los relacionamientos entre "compañeros"... (Foucault, 1983: 249)

Consideramos que el principio de relacionamiento del poder que menciona Foucault, justamente impide la igualación entre capacidad y relación social. Dicha equivalencia trazada por Castells termina por conducir hacia una visión unipolar de las relaciones de poder. Lo que aquí denominamos visión unipolar ${ }^{18}$ de las relaciones de poder, podría igualmente formularse como la visión no relacional de las relaciones sociales y de poder. Una primera demostración del modo que opera esta visión, la podemos registrar en la d1, cuando Castells hace referencia al "aseguramiento de la dominación de una particular relación social de clase" (Castells, 1969a: 148). ¿Cómo es esto de asegurar la dominación de una relación social de clase? ¿Dominación de una relación social o más bien dominación de un actor? ¿El "que" de la dominación es en este caso la relación social? Aquí Castells confunde "dominación de clase", que emplea a menudo en su trabajo, con "dominación de una relación social". En el esquema marxiano del autor, ¿quién sería el "dominador" si no es una clase social? Hay que tener en cuenta que Castells habla de dominación de una relación social de clase y no dominación sobre una relación social. Podemos reconocer este equívoco al introducir otras referencias que aluden a la misma expresión. Por ejemplo: "El aparato jurídicopolítico tiende a asegurar la dominación de las clases dominantes..." (Castells,

\footnotetext{
${ }^{18}$ Cuando decimos "unipolar" damos por supuesto la existencia de una noción de relación social que vincula o pone en relación diferentes polos. Empleamos además la noción de unipolar y no la de unidireccional, porque entendemos que las direcciones pueden eventualmente cambiar aún si circunscribimos la totalidad de la actividad de la relación social, para cada situación, a un solo polo.
} 
1972a: 249). Vemos aquí como Castells habla de asegurar la dominación de las clases dominantes, y no la dominación de una relación social de clase, como en la d1. Si hasta aquí podríamos suponer que el primer caso es producto de un error de Castells antes que de una visión premeditada, esta posibilidad se evapora por completo a partir de constatar la equivalencia directa que establece entre los conceptos de capacidad y relación social, que propone en d5, que es precisamente la definición más detallada del poder. A ello se suman ciertas equivalencias indirectas, como la que registramos en $\mathrm{d} 7$, respecto al vínculo conceptual entre capacidad política y correlaciones de fuerzas (como veremos en el próximo punto). Recordemos entonces que la visión unipolar de las relaciones de poder se efectúa principalmente a partir de la operación teórica de igualación de los conceptos de poder, capacidad y relación social.

\subsubsection{La capacidad política}

En d5 y d7, ambas definiciones contenidas en Luchas de clases en Chile, Castells hace referencia a la noción de capacidad política. Resaltando el concepto, en d5 Castells alude a "la capacidad política de cada clase para realizar sus intereses económicos estructurales" (1974a: 151), mientras que en d7, desplegando su razón dialéctica, hace referencia a la "correspondencia recíproca y de sentido inverso entre la capacidad política del movimiento popular y la de la burguesía" (1974a: 397). En el primer caso la referencia es directa, asociado al núcleo duro de la definición de poder, y en la segunda adopta una forma más bien indirecta. Atendiendo a la similitud del fragmento de la d5 con las restantes definiciones de poder, nos preguntamos: ¿toda capacidad de clase que define el concepto de poder es capacidad política? Partiendo de la primera definición del concepto de poder en 1969, ¿sería correcto hablar de capacidad política de realización de intereses, así como luego, de capacidad política de organización social? ¿El poder es en primera instancia una capacidad política? ¿O se trata más bien de una expresión conceptualmente acotada al libro mencionado? Si es que la referencia política es inherente a las demás nociones de capacidad que acompañan las restantes definiciones de poder, ¿por qué se omite? Por otra parte, la cita de la d5 nos sugiere una nueva consideración de la relación del poder y la capacidad con la política y la economía. La sujeción de Castells al marxismo indica que la referencia a la "realización de intereses" en sus definiciones de poder, refieren en última instancia a la realización de intereses económicos, tal como sugiere para el caso de la d5. Por lo tanto, en principio, la determinación en última instancia de lo económico regiría también para el propio concepto de capacidad. ¿Podríamos decir directamente que el poder, entendido tanto como capacidad de realización o como capacidad de organización, es una capacidad en última instancia económica? En ese caso hablaríamos del poder como capacidad de realización económica, o como capacidad -política- de organización económica. ¿Habremos arribado a estas alturas a la definición del poder como capacidad política de realización o desarrollo económico?

En el caso de la d7, queda claro que si el poder es capacidad política, éste es potencialmente y/o simultáneamente tanto de la burguesía como del movimiento popular. Dicha confirmación vuelve a sembrar controversias respecto a los alcances del concepto de poder. De todos modos, este modo de distribución del poder y la capacidad política cambia radicalmente en las definiciones de poder a 
partir del próximo período, a partir de la desaparición de los movimientos populares como actores capaces políticamente (o políticamente capaces). Representará incluso uno de las operaciones teóricas generales de la definición del poder de Castells.

Finalmente, y relacionado con el apartado anterior, si capacidad es capacidad política, ¿cómo es que una capacidad política es en si una relación social? Aquí quedaría en evidencia, de un modo mas exagerado, el error de pretender igualar poder, capacidad y relación social. Si el poder fuese una relación social, ¿no se tendría que definir entre las capacidades políticas del movimiento social y el de la burguesía, tal como ocurre en la d7?

\subsubsection{La capacidad progresiva}

Finalmente, sólo a modo de mención, podemos constatar que la d8 es la primera definición en la que aparece un registro expansionista del poder. Hace referencia a éste como capacidad de organización para el aseguramiento progresivo de la realización de los intereses específicos de clase (1974a: 361). Ello nos permitiría rescatar una cierta noción de capacidad progresiva, que si bien Castells no formula en esos términos, en ningún momento contradice. Más adelante, en un próximo trabajo, podremos constatar la necesidad de esta breve mención, ya que nos permitirá abonar el terreno para las comparaciones con los períodos posteriores (1983-2003; 2004-2009).

\section{A MODO DE SÍNTESIS}

Organizamos las conclusiones del trabajo a partir del desarrollo de tres puntos, que representan en gran medida los tópicos principales del método de nuestra investigación general, aplicados en este caso a la relación entre los conceptos de poder y capacidad en las definiciones de poder de este primer periodo de Manuel Castells. Hablamos de las operaciones teóricas principales, los movimientos de cambio conceptual y las concepciones del poder. Veamos cada uno de ellos:

a) Las operaciones teóricas principales: Antes de entrar en detalles, es menester reconocer una suerte de operación teórica general que efectúa Castells sobre este relación específica -aunque no sólo sobre ella-: la explicitación selectiva de la fuente teórica de su definición del poder. Pese al empleo sistemático de la definición de Poulantzas en todas sus definiciones, tan sólo en dos de ellas (d2, d6) Castells reconoce la autoría de aquel. ¿Por qué omitir parcialmente a Poulantzas? Si bien por el momento no tenemos respuestas ni ensayos de respuesta a esta pregunta, consideramos que no es un hecho menor. Volvamos a la propuesta específica de este punto.

A lo largo del período que transcurre entre 1969 y 1982, en principio identificamos seis operaciones teóricas que efectúa Castells en relación al vínculo entre poder y capacidad:

1. La no definición de la noción de capacidad.

2. La eventual igualación de los conceptos de capacidad y relación social, o bien de poder, capacidad y relación social 
3. La contraposición entre capacidad de organización y simple capacidad coercitiva.

4. La omisión del grado o tipo de participación de la coerción o la violencia en la nueva formula organizacional del poder.

5. La omisión de la definición de organización social de Poulantzas como "condición de poder".

6. La reducción de la capacidad del poder a una capacidad política.

De las seis operaciones presentadas, la más importante posiblemente sea la $n^{\circ} 2$ : ésta permite el desarrollo de una visión unipolar de las relaciones de poder, tal como afirmamos en el cuerpo del trabajo.

b) Los movimientos de cambio conceptual: Se relacionan directamente con buena parte de las operaciones mencionadas arriba. En este segundo punto distinguimos entre los movimientos de cambio conceptual en relación a los textos propios, y aquellos movimientos que se producen en relación a las fuentes teóricas de Castells, intrínseco al proceso de apropiación teórica. A partir de una mirada diacrónica a los textos del propio Castells, detectamos básicamente un solo movimiento general de cambio: el paso del poder como capacidad de realización de intereses específicos, mayoritariamente presente en La cuestión urbana, al poder como capacidad de organización social para la realización de intereses específicos, principalmente expresado en Lucha de clases en Chile. No estamos en condiciones de establecer una hipótesis concluyente respecto a los motivos de dicho movimiento. Dado los elementos que disponemos sólo se nos ocurre pensar que a partir de la identificación explícita entre poder y organización social, Castells busca inscribirse en un concepto más amplio de poder, priorizando -como ya hemos señalado- una idea de capacidad organizativa que incluye al conjunto de lo social, opuesta al poder como capacidad coercitiva, y probablemente a cualquier expresión reduccionista de clase. Pero esta suposición es débil, en la medida que constatamos que la definición previa de poder de Castells, en su formulación general, es prácticamente la misma que emplea Poulantzas, y a partir de la cual éste último identifica capacidad con organización social. Igualmente, Castells no ofrece ninguna pista que nos permita suponer que aceptó esta última acepción de capacidad.

En segundo lugar, tal como venimos repitiendo -esperamos no agotar al lector-, las fuentes teóricas de la definición del concepto de poder de Castells prácticamente se circunscriben a un solo autor: Nicos Poulantzas. Respecto a éste, y en relación al vínculo entre poder y capacidad, identificamos principalmente tres movimientos específicos:

1. El paso de la definición de la noción de capacidad como organización (Poulantzas) a su no definición.

2. De la diferenciación de los conceptos de capacidad y relación social (Poulantzas) a su igualación.

3. De la definición de organización social como condición de poder (Poulantzas) a su completa omisión.

Podemos observar que el punto 3 cobra especial sentido en relación al punto 1 , ya que lo que Castells finalmente omite en su discurso es el reconocimiento de la capacidad como condición de poder, lo cual no es equivalente al poder como un 
todo. Este hecho es fundamental, ya que tal reconocimiento permitiría precisar y limitar los alcances de la noción de capacidad en relación al poder, rompiendo con la igualación entre ambos conceptos, a favor de una idea más amplia de poder, definida en primera instancia pero no completamente determinada a partir de la capacidad.

c) Las concepciones del poder: En este último apartado pretendemos sintetizar nuestra interpretación sobre aquellos aspectos de la concepción del poder de Castells que se expresan a partir de la relación específica propuesta. Partiendo de la relación existente y explicitada entre los diferentes elementos de las definiciones, nos permitiremos sugerir ciertas pistas para nuevas interpretaciones, que si bien en todo momento se ajustan a los términos del autor, desarrollan un discurso hipotético sobre algunos sentidos no explicitados. Con ello podríamos completar las definiciones del poder de Castells en este primer período, en lo que respecta a la relación específica propuesta.

En el eje que vincula poder y capacidad, constatamos que Castells define el poder atendiendo a tres problemas centrales: 1 . La relación entre poder y violencia; 2. La relación entre poder y organización; 3 . El status político de la capacidad. En el primer caso, Castells define el poder como capacidad de organización, en oposición y/o como formula superadora de la noción de poder como "simple atribución de capacidad coercitiva" (d6). Si bien todo indica que la primera se integra en la segunda, Castells no precisa el modo de participación de la coerción en la nueva formula organizacional del poder. Ello nos remite directamente a la relación entre poder y violencia. Es importante señalar que el rechazo a la identificación plena entre poder y coerción, que el autor reconoce como una limitación de las teorías liberal e histórica del poder, es un posicionamiento exclusivo de este primer periodo (1969-1982). En el segundo caso, en contraste con Poulantzas, para Castells el concepto de capacidad no equivale a organización social (capacidad como organización), sino que se agrega a ésta, en la forma de capacidad de organización. Por lo tanto no queda claro que entiende exactamente por capacidad. Con ello se haría evidente un principio de redundancia en Castells ${ }^{19}$, que incrementa la opacidad que acompaña su definición de poder. En tercer lugar, como ya insinuamos en el trabajo, desde la visión de Castells es posible considerar toda capacidad de clase que se desprende de la definición de poder como una capacidad política. Ello contraviene la definición de capacidad de Poulantzas, de carácter multidimensional. Si para Castells capacidad es en todo momento capacidad política, podríamos hablar tanto de capacidad política de realización de intereses, así como luego, de capacidad política de organización social. Ahora bien, siguiendo la d7, el poder como capacidad política es potencialmente y/o simultáneamente tanto de la burguesía como del movimiento popular, lo cual en principio se contradice con la identificación del poder con las fuerzas del cambio social. Llegado a este punto, dado que el concepto de capacidad política se desarrolla al mismo nivel de concreción que el de movimiento social, es necesario reconocer el uso simultáneo, en un nivel concreto, de dos ideas contrapuestas de la relación entre poder y clases sociales: la que define el poder como propiedad exclusiva del proletariado, representando las fuerzas de cambio social, y la que entiende el

\footnotetext{
${ }^{19} \mathrm{Si}$ bien no es el objetivo preciso de nuestro trabajo, siempre es difícil ampliar el análisis comparativo de las diferencias entre Castells y Poulantzas, ya que el primero no argumenta sus elecciones conceptuales al mismo nivel que el segundo.
} 
poder como capacidad política de cualquier clase. Los contrapuntos y las diferencias sustanciales con Poulantzas son un hallazgo espinoso en la medida que Manuel Castells en ningún momento reconoce explícitamente las diferencias con aquel, sino más bien todo lo contrario: asume el discurso (y no la posición) de aquel hijo obediente que aún goza y elige la protección del padre.

\section{BIBLIOGRAFÍA}

(1967) CASTELLS, Manuel, "Mobilité des entreprises et structure urbaine", Sociologie du Travail, 4, pp. 369-405.

(1968a) CASTELLS, Manuel, Metodología de la práctica sociológica, FlacsoElas-Elacp, Santiago de Chile.

(1968b) CASTELLS, Manuel, "Is there an urban sociology?". En C.G Pickvance (ed.,1976), Urban Sociology: Critical Essays, St.Martin Press, New York,

(1968c) CASTELLS, Manuel, "La mobilité des entreprises industrielles dans la région Parisiense", Cahiers de l'Institut d'Aménagement et d'Urbanisme de la Région Parisienne, Vol. 11.

(1969a) CASTELLS, Manuel, "Theoretical propositions for an experimental study of urban social movement". En C.G Pickvance (ed.,1976), Urban Sociology: Critical Essays, St. Martin Press, New York, pp.147-173.

(1969b) CASTELLS, Manuel, "Theory and ideology in urban sociology". En C.G Pickvance (ed.,1976), Urban Sociology: Critical Essays, St.Martin Press, New York, pp.60-84.

(1969c) CASTELLS, Manuel, "Towards a Sociological Theory of City Planning". En Charles Lemert (ed.,1981), French sociology. Rupture and renewal since 1968, New York, Columbia University Press, pp. 374-396.

(1969d) CASTELLS, Manuel, "Le centre urbain. Project de recherche sociologique". Cahiers internationaux de sociologie 46: 83-106.

(1969e) CASTELLS, Manuel, "Entreprise industrielle et développment urbain". Synopsis, September, pp.69-79.

(1969f) CASTELLS, Manuel, "Vers une théorie sociologique de la planification urbaine", Sociologie du Travail, 4, pp.130-143.

(1970a) CASTELLS, Manuel, "Structures sociales et processus d'urbanisation: analyse comparative intersociétale", Annales: économies, sociétés, civilizations. Numéro spécial Histoire et urbanisation, Vol. 25, 4, juillet-août, pp. 1155-1199.

(1970b) CASTELLS, Manuel; et al., "Réconquete urbaine et rénovationdéportation a Paris", Sociologie du Travail 4, pp. 488-514.

(1970c) CASTELLS, Manuel, "La renovation urbaine aux Etats-Unis", Espaces et Sociétés, 1, pp. 107-137.

(1971a) CASTELLS, Manuel, Problemas de investigación en sociología urbana, Siglo XXI, Madrid. 
(1971b) CASTELLS, Manuel. "La sociologie et la question urbaine". L'architecture d'aujourd'hui, Septembre, pp. 91-100.

(1971c) CASTELLS, Manuel, "El mito de la sociedad urbana", Eure: Revista Latinoamericana de Estudios Regionales, Vol.1, 3, Octubre.

(1972a) CASTELLS, Manuel, La cuestión urbana, México: Siglo XXI, 2004.

(1972b) CASTELLS, Manuel, "Luttes de classes et contradictions urbaines", Espaces et Societes, October, 6-7.

(1972c) CASTELLS, Manuel, "Symbolique urbaine et mouvements sociaux", Versus: Quaderni di Studi Semiotici

(1972d) CASTELLS, Manuel, "Las nuevas fronteras de la metodología sociológica", Revista Latinoamericana de Ciencias Sociales, 3, Junio.

(1972e) CASTELLS, Manuel, "Urban Renewal and Social Conflict in Paris", Social Science Information, Vol. 11, 2, April, pp. 93-124.

(1973a) CASTELLS, Manuel (comp.), Imperialismo y urbanización en América Latina, Gustavo Gili, Barcelona.

(1973b) CASTELLS, Manuel; et. al., La rénovation urbaine à Paris: structure urbaine et logique de classe, Mouton, Paris.

(1973c) CASTELLS, Manuel, "Comentario: La teoría marxista de las clases sociales y la lucha de clases en América Latina". En Raúl Benitez Zenteno (ed.), Las clases sociales en América Latina, Siglo XXI, México.

(1973d) CASTELLS, Manuel, "La urbanización dependiente en América Latina", M. Castells, Manuel; et. al. (eds.), Imperialismo y urbanización en América Latina, Gustavo Gili, Barcelona, 7-26.

(1973e) CASTELLS, Manuel, "Il rinovo urbano di Parigi: aspetti economici e politici", Archivio di Studi Urbani e Regionali, 2.

(1973f) CASTELLS, Manuel, "Movimiento de pobladores y lucha de clases en Chile", Revista Latinoamericana de Estudios Urbanos, 3.

(1973g) CASTELLS, Manuel, "Tesi sulla questione urbana", Archivio di Studi Urbani e Regionali, 1.

(1974a) CASTELLS, Manuel, La lucha de clases en Chile, Siglo XXI, Argentina.

(1974b) CASTELLS, Manuel; GODART, Francis, Monopolville: l'entreprise, l'état, l'urbain, Paris, Mouton.

(1974c) CASTELLS, Manuel (ed.), Estructura de clases y política urbana en América Latina, Sociedad Interamericana de Planificación, Buenos Aires.

(1974d) CASTELLS, Manuel, "Contraddizioni e diseguaglianza nella città", II Mulino, 1.

(1974e) CASTELLS, Manuel, "Consommation collective, intérêt de classe et processus politique dans le capitalisme avancé", Papers: Revista de Sociologia, 3, 63-89.

(1975a) CASTELLS, Manuel, Sociología del espacio industrial, Ayuso, Madrid.

(1975b) CASTELLS, M.; A. Pizzorno; y M. Kaplan (eds.), Participación y cambio social en la problemática contemporánea, Siap-Planteos, Buenos Aires. 
(1975c) CASTELLS, Manuel, "Advanced Capitalism, Collective Consumption, and Urban Contradictions: New Sources of Inequality and New Models for Change". En Leon Lindberg, et. al., (ed.), Stress and Contradiction in Modern Capitalism, Lexington Books, Toronto, pp.175-197.

(1975d) CASTELLS, Manuel, "Squatters and Politics in Latin America: A comparative Analysis of Urban Social Movements in Chile, Peru and Mexico". En Helen Safa (ed.,1982), Towards a Political Economy of Urbanization in Third World countries, Oxford University Press, Stanford, pp. 249-282

(1975e) CASTELLS, Manuel, "La fonction sociale de la planification urbaine: le cas de la région de Dunkerque", Recherches Sociologiques, 3.

(1975f) CASTELLS, Manuel, "Immigrant Workers and Class Struggles in Advanced Capitalism: the Western European Experiencie", Politics Society, 5.

(1976a) CASTELLS, Manuel, La crisis económica mundial y el capitalismo americano, Laia, Barcelona.

(1976b) CASTELLS, Manuel, "Crisis del Estado, Consumo colectivo y Contradicciones Urbanas". En Nicos Poulantzas (ed., 1977), La crisis del Estado, Fontanella, Barcelona, pp. 205-237.

(1976c) CASTELLS, Manuel, "La nueva estructura de la dependencia y los procesos políticos de cambio social en América Latina". En Alejandro Pizzorno (ed.), Participación y cambio social en la problemática contemporánea, SIAP, Buenos Aires.

(1976d) CASTELLS, Manuel, "The wild city". En Feagin, Joe R. (ed., 1979), The Urban Scene. Myths and Realities, Random House, New York.

(1976e) CASTELLS, Manuel, "The Service Economy and the Post-Industrial Society. A Sociological Critique", International Journal of Health Services, 4, Vol. 6, pp. 596-607.

(1976f) CASTELLS, Manuel, "La crise urbaine aux Etats-Unis: vers la barbarie?", Temps modernes 355, pp.1177-1240.

(1976g) CASTELLS, Manuel; DE IPOLA, Emilio, "Epistemological practice and the social science", Economy and Society, 2, Vol. 5, May.

(1977ª) CASTELLS, Manuel, Ciudad, Democracia y Socialismo, Siglo XXI, Madrid.

CASTELLS, Manuel (1977b) "Towards a Political Urban Sociology". En Michael Harloe (ed.), Captive cities. Studies in the Political Economy of Cities and Regions, John Wiley \& Sons, London, pp. 61-78.

(1977c) CASTELLS, Manuel, "Marginalité urbaine et mouvements sociaux au Mexique: le mouvement des 'posesionarios' dans la ville de Monterrey", International Journal of Urban and Regional Research, Vol. 1, 1, Mars, pp.145150.

(1977d) CASTELLS, Manuel, "Les conditions sociales d'émergence des mouvements sociaux urbains (à partir d'une enquête exploratoire sur les luttes urbaines dans la région Parisienne, 1968-73)". International Journal of Urban and Regional Research, Vol. 1, 1, 45, Mars. 
(1977e) CASTELLS, Manuel, "Apuntes para un análisis de clase de la política urbana del Estado mexicano", Revista Mexicana de Sociología, Vol. 39, 4, pp. 1161-1191.

(1977f) CASTELLS, Manuel; POULANTZAS, Nico, "Débat avec Christine BuciGlucksmann", Les Cahiers du Ceres, Janvier, pp. 11-35.

(1978a) CASTELLS, Manuel, La teoría marxista de las crisis económicas y las transformaciones del capitalismo, Siglo XXI, Madrid.

(1978b) CASTELLS, Manuel, City, class and power, Mac Millan, London.

(1978c) CASTELLS, Manuel; et al., Crise du logement et mouvements sociaux urbains. Enquête sur la région Parisienne, Mouton, Paris.

(1978d) CASTELLS, Manuel "Urban Social Movements and the Struggle for Democracy: the Citizens Movement in Madrid". International Journal of Urban and Regional Research, Vol. 2, 1, pp. 133-146.

(1978e) CASTELLS, Manuel, "Social Organization and Social Movements in Latin American Squatter Settlements". Ponencia preparada para el panel on Third World Urbanization held at the Xth International Congress of Anthropological and Ethnological Sciences, Diciembre, Nueva Delhi, India.

(1979a) CASTELLS, Manuel, "Revisar a Engels", Argumentos, Julio.

(1979b) CASTELLS, Manuel, "La intervención administrativa en los centros urbanos de las grandes ciudades", Papers: Revista de Sociologia, 11, pp. 227250.

(1980) CASTELLS, Manuel, "Cities and Regions Beyond the Crisis: Invitation to a Debate". International Journal of Urban and Regional Research, Vol, 4, 1, March.

(1981a) CASTELLS, Manuel, Capital multinacional, estados nacionales $y$ comunidades locales, Siglo XXI, México.

(1981b) CASTELLS, Manuel, Crisis urbana y cambio social, Siglo XXI, Madrid.

(1981c) CASTELLS, Manuel, "Local government, urban crisis, and political change". En Maurice Zeitlin (ed.), Political Power and Social Theory, Vol. 2, JAI Press, Stanford, pp.1-19.

(1981d) CASTELLS, Manuel, "Housing Policy and Urban Trade Unionism: the Grands Ensambles of Paris". Informe de investigación (Working paper; 363), Berkeley, University of California: Institute of Urban and Regional Development.

(1981e) CASTELLS, Manuel. "Community Participation, Planning, and the State in the Metropolitan Areas of Developing Countries", Informe de investigación: Institute of Urban and Regional Development, University of California, Berkeley.

(1982a) CASTELLS, Manuel, "Mouvements sociaux urbains et changement politique". En Alain Touraine, Mouvements sociaux d'aujourd'hui: acteurs et analystes, Les Éditions Ouvrières, Paris, pp. 201-207.

(1982b) CASTELLS, Manuel; Karen Murpy, "Cultural Identity and Urban Structure: The Spatial Organization of San Francisco's Gay Community". En Norman Fainstein; Susan Fainstein (eds.), Urban Policy under Capitalism, London, pp. 237-259. 
(1982c) CASTELLS, Manuel, "Planning and Social Change: Introduction", Journal of Planning Education and Research, Vol. 2, 3.

(1998) CHIGNOLA, Sandro, "Historia de los conceptos e historiografía del discurso político", Res Publica, 1, 1988, pp. 7-33.

(1983) FOUCAULT, Michel, "El sujeto y el poder". En Hubert Dreyfous; Paul Rabinow (eds., 2001), Michel Foucault: Más allá del estructuralismo y la hermenéutica, Nueva Visión, Buenos Aires, pp. 241-259.

(2004) GARNHAM, Nicholas, "Information Society Theory as Ideology". En: Webster, F. (ed.), The Information Society Reader, Routledge, New York.

(1984) GIDDENS, Anthony, "La constitución de la sociedad. Bases para una teoría de la estructuración", Amorrortu, Buenos Aires.

(2003) KELLNER, Douglas, Theorizing Globalization, Essay UCLA, U.S.

(1998) MERLO, Mauricio, "La ambivalencia de los conceptos. Observaciones acerca de algunas relaciones entre Begriffsgeschichte e historiografía del discurso político", Res Publica, 1, pp. 87-101.

(1968) POULANTZAS, Nicos, Poder político y clases sociales en el estado capitalista, Siglo XXI, México.

(2004) SIERRA CABALLERO, Francisco, "El mercado y sus marcas: Los nuevos territorios y contradicciones teóricas de la investigación en comunicación", Revista Latinoamericana de Comunicación, Octubre.

(2008) TORRES CASTAÑOS, Esteban, "Ciencias Sociales, Historia de los conceptos y la idea de Trayectoria conceptual", RIS: Revista de Investigación Social, 4, 7, Invierno, UNAM, México, pp. 81-101.

(2003) TREMBLAY, Gaetan, "La sociedad de la información y la nueva economía. Promesas, realidades y faltas de un modelo ideológico", TELOS, 54, Segunda época, Madrid.

VAN DIJK, Jan. (1999) The One-Dimensional Network Society of Manuel Castells. New Media Society 1999, 1, 127

(2004) WEBSTER, Frank.; BLOM, Raimo, (eds.), The Information Society Reader, Routledge, U.S. 